\title{
Tuberous Sclerosis Associated with Multiple Hepatic Lipomatous Tumors and Hemorrhagic Renal Angiomyolipoma
}

\author{
Shoji Hirasaki, Norio KoIde, Hiromichi Ogawa*, Kozo Ujike, Toshiyuki Shinj and Takao TsujI \\ We report a case of tuberous sclerosis associated with hepatic lipomatous tumors and renal \\ angiomyolipomas. Abdominal ultrasonography revealed a high echoic large tumor in the left kid- \\ ney. A provisional diagnosis of angiomyolipomas of the kidney was made based on computed \\ tomography. Subsequent laparotomy revealed that the extracted tumor was renal angiomyolipoma. \\ It was also revealed that there was an association with hepatic lipomatous tumors thought to be \\ lipomas or angiomyolipomas by liver biopsy. Nearly half of all cases of angiomyolipoma in the \\ kidney are reported as occasional association with tuberous sclerosis complex, but lipomatous \\ tumors in the liver are rare. \\ (Internal Medicine 38: 345-348, 1999)
}

Key words: hepatic hamartoma, hepatic angiomyolipoma, ultrasonography-guided liver biopsy, renal hamartoma, renal hemorrhage

\section{Introduction}

Tuberous sclerosis is a rare heredofamilial disease characterized by a variety of hematomatous lesions in the brain, skin, retina, and viscera. Angiomyolipoma, composed of mixed mesenchymal tissue, is not uncommon in the kidney, and many cases of renal angiomyolipoma are associated with other malformations as part of the syndrome of tuberous sclerosis. However, hepatic multiple lipomas or angiomyolipomas are rarely encountered. A diagnosis of renal angiomyolipoma or hepatic lipomatous tumor propounds considerable problems as the entity is difficult to distinguish from malignant tumor (1-3) and in some cases, it is found only after rupture, bleeding and the detection of disseminated intravascular coagulation $(4,5)$. Herein we report the case of a Japanese woman diagnosed with hepatic multiple lipomatous tumors supposed to be lipomas or angiomyolipomas and hemorrhagic renal angiomyolipoma associated with tuberous sclerosis.

\section{Case Report}

The patient was a 30 -year-old Japanese woman, $156 \mathrm{~cm}$ tall and weighing $53.0 \mathrm{~kg}$. She was admitted to our hospital complaining of left flank pain and grosshematuria of several days duration. She was diagnosed with tuberous sclerosis at the age of 6 years. She is mentally retarded and has a long history of generalized grand mal seizures, which have been controlled with phenobarbital. A family history of a neurocutaneus syndrome could not be elicited.

The patient's body temperature was $37.0^{\circ} \mathrm{C}$, blood pressure was $130 / 96 \mathrm{mmHg}$, and radial pulse rate was $78 / \mathrm{min}$ and regular. She was noted as having adenoma sebaceum in a butterfly paranasal distribution. She had neither anemia nor jaundice. Neurological examination revealed no abnormal findings except for mild mental retardation. Abdominal palpation revealed a mass with tenderness in the left upper quadrant.

Laboratory findings showed a red blood cell count of $384 \times 10^{4} / \mathrm{mm}^{3}$, a white blood cell count of $5,200 / \mathrm{mm}^{3}$, and a platelet count of $24.8 \times 10^{4} / \mathrm{mm}^{3}$. The hemoglobin concentration was $10.0 \mathrm{~g} / \mathrm{dl}$. The liver function tests revealed: asparate aminotransferase, $12 \mathrm{IU} / l$; alanine aminotransferase, $5 \mathrm{IU} / l$; alkaline phosphatase, $234 \mathrm{IU} / l$; leucin amino peptidase, $59 \mathrm{IU} /$ $l ; \gamma$-glutamyltranspeptidase, 147 IU/l (normal 8-48); cholinesterase, $196 \mathrm{IU} / l$; lactate dehydrogenase, $389 \mathrm{IU} / l$; and total bilirubin, $0.4 \mathrm{mg} / \mathrm{dl}$. Tests for $\mathrm{C}$ reactive protein revealed 0.11 $\mathrm{mg} / \mathrm{dl}$, and the erythrocyte sedimentation rate was $9 \mathrm{~mm} / \mathrm{h}$. With respect to renal function, blood urea nitrogen was $11.0 \mathrm{mg} / \mathrm{dl}$ and creatinine was $0.7 \mathrm{mg} / \mathrm{dl}$. Serological studies for hepatitis $\mathrm{B}$ and $\mathrm{C}$ viruses were negative. Electroencephalogram revealed no remarkable findings and a brain computed tomography (CT) scan showed multiple calcification.

Ultrasonography (US) performed by a urologist revealed a

From the First Department of Internal Medicine, Okayama University School of Medicine, Okayama and *the Department of Internal Medicine, Kagawaken Saiseikai Hospital, Takamatsu

Received for publication June 1, 1998; Accepted for publication January 14, 1999

Reprint requests should be addressed to Dr. Shoji Hirasaki, the Department of Internal Medicine, Kagawaken Saiseikai Hospital, 1-16-4 Sakuramachi, Takamatsu $760-0074$ 
large, highly echoic, heterogeneous tumor around the left kidney, measuring $132 \times 84 \mathrm{~mm}$. An abdominal CT scan revealed a large, heterogeneous mass around the left kidney and small, round, low-density tumors in the right kidney (Fig. 1). An abdominal CT also showed small, round, multiple, low-density tumors in the bilateral lobes of the liver (Figs. 1,2). The clinical diagnoses, based on these imaging examinations, were angiomyolipomas of the kidney and cysts in the liver.

The patient underwent a laparotomy because of the continuation of abdominal pain and progress of anemia after admission. During surgery, the soft tumor with yellowish-gray cut surface was found occupying the left side of the abdominal cavity. Left nephrectomy was performed and the left renal tumor was extracted at the same time. The histological diagnosis of renal tumor was angiomyolipoma. As a result of this treatment the patient's abdominal pain and gross hematuria were resolved and anemia was improved postoperatively.

The patient visited our department after the operation for a thorough examination. An abdominal US revealed that there were multiple high echoic round tumors with acoustic shad-
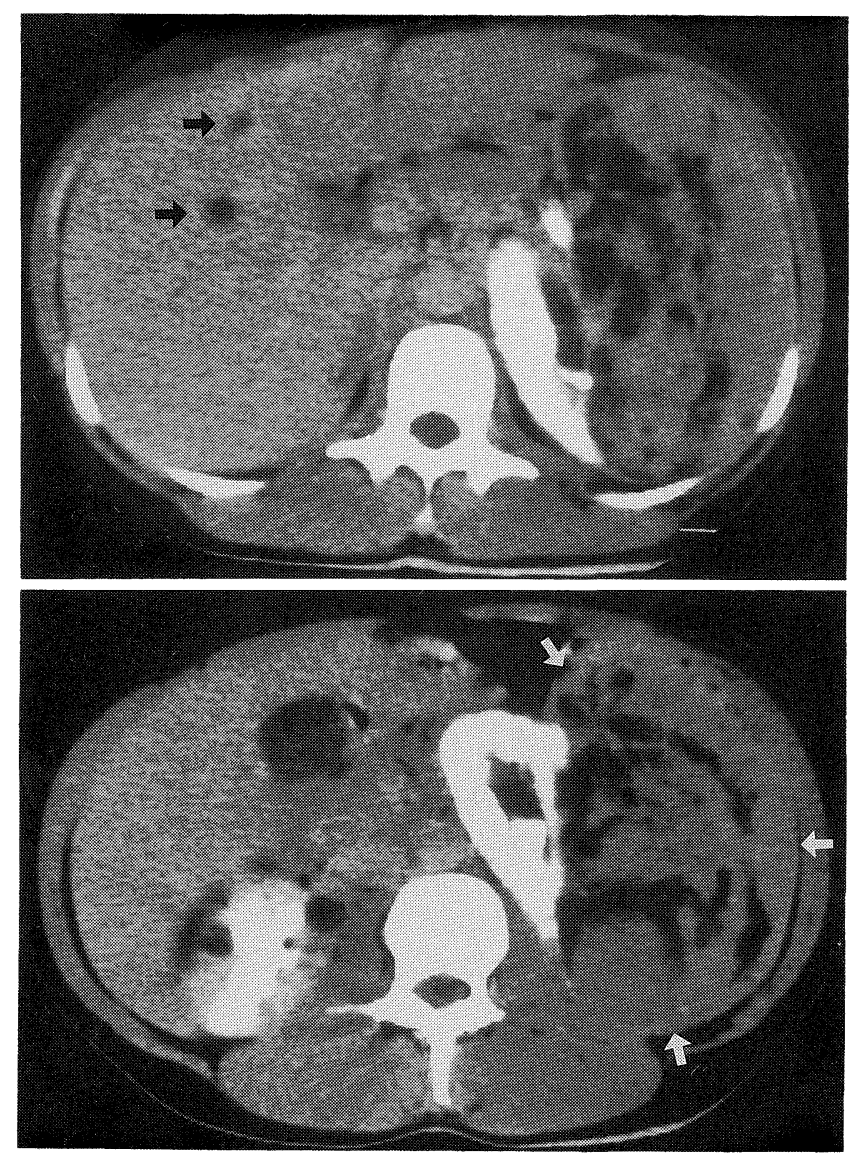

Figure 1. Image obtained by abdominal CT scan through kidney. CT revealed a large, heterogenious mass in the left kidney (white arrows) and small round low-density tumors in the right kidney. It also revealed round, multiple, low-density tumors in the liver (black arrows). ows in the liver (Fig. 3), and no cysts were seen. These observations suggested that the tumors preoperatively detected in the liver were not cysts and the diagnosis should be changed to lipoma or angiomyolipoma of the liver. Subsequent dynamic CT showed that the multiple liver tumors had contrast enhancement (Fig. 2). These imaging examinations suggested that the liver tumors were more likely to be angiomyolipoma than lipoma. On the basis of this diagnosis we performed ultrasonog-
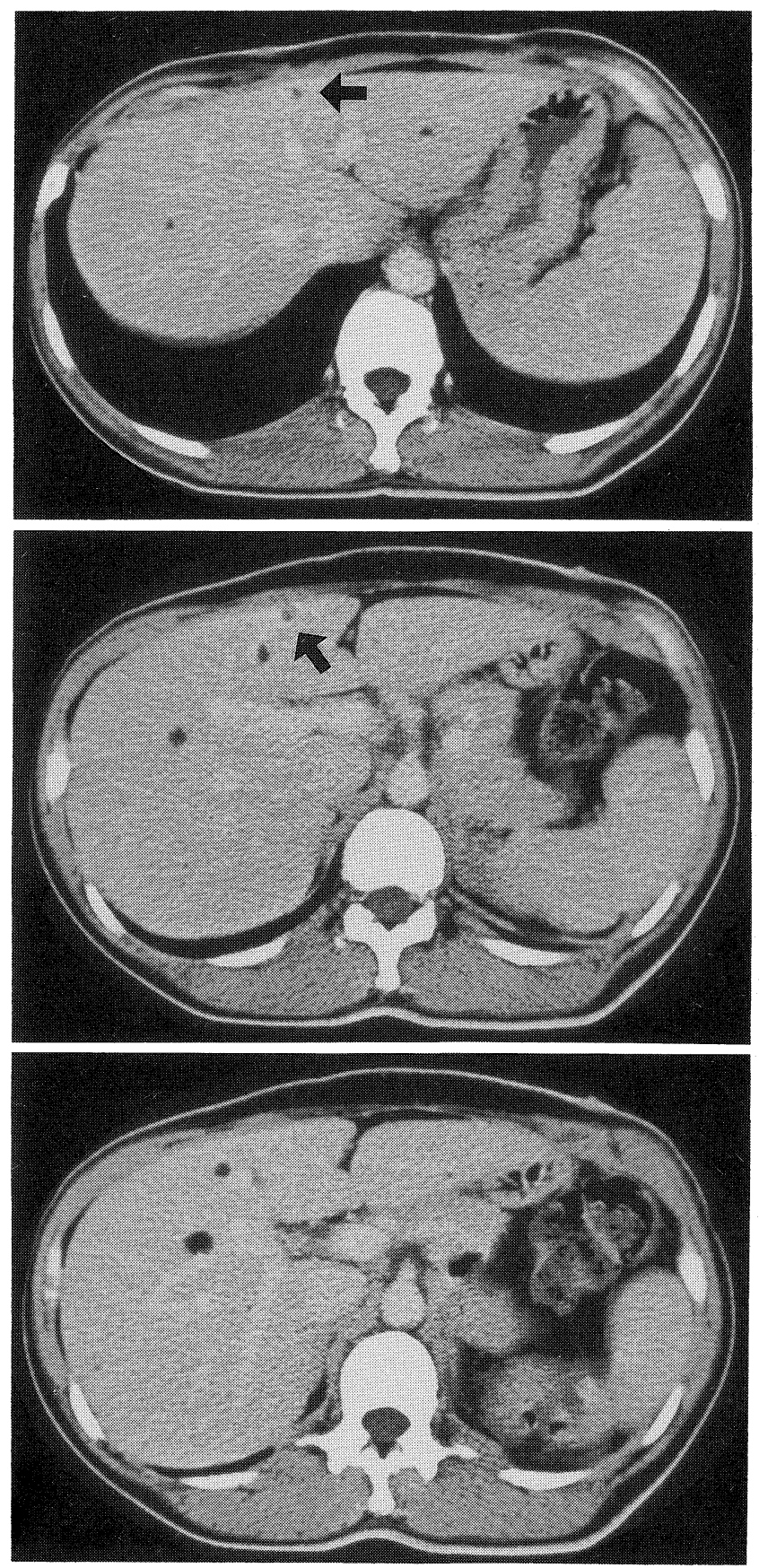

Figure 2. Images obtained by abdominal spiral CT scan through liver after left nephrectomy. Dynamic CT showed the multiple liver tumors with contrast enhancement (arrows). 

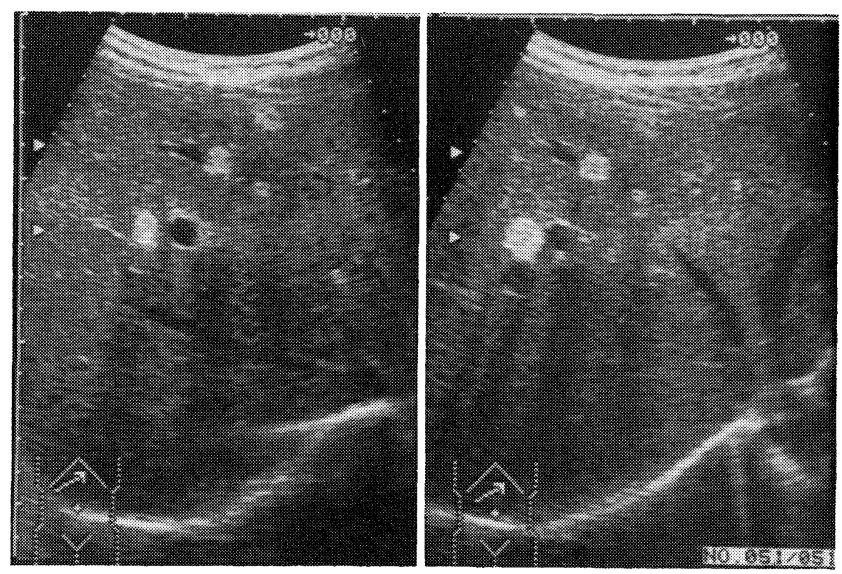

Figure 3. Images of abdominal US.Abdominal US revealed numerous multiple high echoic round tumors with acoustic shadows in the liver.

raphy-guided liver biopsy. A liver biopsy specimen appeared to have only adipose tissue, resulting in a diagnosis of hepatic lipomatous tumor. We could not make a definite diagnosis of lipoma or angiomyolipoma by histological examination.

\section{Discussion}

Tuberous sclerosis is an autosomal dominant disorder characterized by adenoma sebaceum, epilepsy, and mental retardation. It is phacomatosis with a strong preponderance in females (6-8). Patients often have renal involvement with angiomyolipoma. Renal angiomyolipoma in patients with tuberous sclerosis is usually multiple, bilateral and asymptomatic, and may occur at an early age. Symptoms of renal angiomyolipoma usually result from hemorrhage in or around the tumor. Symptoms include: pain and hematuria, and a significant proportion of patients exhibit shock. A small number of patients will develop renal failure from progressive renal parenchymal replacement by tumor (9). The differential diagnosis for renal angiomyolipoma with or without the tuberous sclerosis complex includes polycystic kidney disease and renal neoplasm. If the stigmata of tuberous sclerosis are present, the diagnosis is clear. However, without the stigmata, it is difficult to distinguish the three lesions since they can present with enlarged kidneys and a benign urinalysis. The classic triad of flank pain, painless hematuria and palpable abdominal mass have been emphasized as being strongly suggestive of the presence of a renal neoplasm, though differentiation from unilateral angiomyolipomas is especially difficult (10).

Recently, preoperative diagnosis of renal angiomyolipoma has become possible with the development of various imaging modalities and the emphasis of treatments of renal angiomyolipoma has shifted to preservation of renal function through partial nephrectomy, enucleation of the tumor and arterial embolization to prevent rupture $(9,11)$. However, a concurrence of renal angiomyolipoma and cancer in the same kidney (12) and sarcomatous changes of angiomyolipoma (13) have been reported. Therefore, a careful diagnosis is important in the conservative treatment of renal angiomyolipoma.

Lipoma or angiomyolipoma is relatively rare in the liver. More than half of all cases are asymptomatic, whereas the others have only fine clinical manifestations such as epigastric or right upper quadrant pain or discomfort (14). Hepatic involvement in tuberous sclerosis has been described in the radiographic and pathologic literature $(15,16)$. Based on the CT findings, multiple liver cysts were suspected at first in the present case. An abdominal US performed after the operation showed hyperechoic multiple tumors in the liver, and supported the clinical diagnosis of multiple hepatic lipoma or angiomyolipoma. In our case, diagnosis of the hepatic lipomatous tumor was established by ultrasonography-guided biopsy and malignant tumor was ruled out. Angiomyolipoma generally consists of different lesions; in adipose tissue, blood vessels and smooth muscle, all of which were barely visible in the specimen obtained by ultrasonography-guided liver biopsy. Although the biopsy specimen showed adipose tissue only, hepatic angiomyolipoma was not completely ruled out in the present case.

Compton et al (15) reported that angiographically, five of eight patients with tuberous sclerosis had vascular tumors in the liver consistent with hamartomas. Roberts et al (17) reported seven cases of lipomatous masses in the liver, five of which were obtained from a retrospective review of 50 cases of renal angiomyolipoma and three of which were associated with tuberous sclerosis. The true incidence of angiomyolipomas or lipomatous tumors in the liver is not known because of the difficulty in detection. The detectability of these lesions is limited by the following facts (17): 1) fatty tumors are rare, 2) fat is difficult to detect in lesions with a low fat content, 3) the lesions are asymptomatic and hence are detected only as incidental findings, 4) small lesions can be confused for a simple cyst due to partial volume averaging, and 5) a peripheral mass or one adjacent to the fat-containing falciform ligament is difficult to appreciate. In fact, the cases of tuberous sclerosis associated with hepatic multiple lipomatous tumors are rare although nearly half of all cases of angiomyolipoma in the kidney are reported as an occasional association with tuberous sclerosis complex $(11,18)$. However, since several cases of tuberous sclerosis associated with hepatic lipoma or angiomyolipoma have been reported (17), some relationship may exist between tuberous sclerosis and hepatic hamartoma in regard to the etiology. Further studies on the relationship between tuberous sclerosis, renal angiomyolipoma, and hepatic lipomatous tumor are required. In our patient, we do not have direct evidence that tuberous sclerosis is related to the development of hepatic lipomatous masses. However, we believed it would be valuable to report this case of tuberous sclerosis associated with renal angiomyolipomas and hepatic lipomatous tumors, since such a disease association is rare.

\section{References}

1) Wong K, Kaisary AV, Waters CM, Horner J, Hershman MJ. 


\section{HiRASAKI et al}

Angiomyolipoma of the kedney: a clinical enigma in diagnosis and management. Ann R Coll Surg Engl 74:144-148, 1992.

2) Kawarada Y, Mizumoto R. Angiomyolipoma of the liver. Am J Gastroenterol 78:434-439,1983.

3) Goodman ZD, Ishak KG. Angiomyolipoma of the liver. Am J Surg Pathol 8: 745-750, 1984.

4) Henslee D, Ross G Jr, Beale G. Bilateral angiomyolipomas: The benefits and limitation of embolization for renal salvage -A case report-. Mo Med 88:292-294, 1991.

5) Ukai K, Fuji A, Nishiwaki J, et al. Giant hepatic angiomyolipoma associated with disseminated intravascular coagulation. Intern Med 36:186190, 1997.

6) Farrow GM, Harrison EG, Utz DC, Jones DR. Angiomyolipoma. A clinicopathologic study of 32 cases. CA 22:564-570, 1968.

7) Bagley D, Appell R, Pingoud E, McGuire EJ. Renal angiomyolipoma diagnosis and management. Urology 25:1-5, 1980.

8) Viamonte M Jr, Ravel R, Politano V, Bridges B. Angiographic findings in a patient with tuberous sclerosis. Am J Roentgenol Radium Ther Nucl Med 98:723-733, 1966.

9) Sanchez FW, Vujic I, Ayres RI, Curry NS, Gobien RP. Hemorrhagic renal angiomyolipoma: superselective renal arterial embolization for preservation of renal function. Cardiovasc Intervent Radiol 8: 39-42,1985.

10) Chonko AM, Weiss SM, Stein JH, Ferris TF. Renal involvement in tuber- ous sclerosis. Am J Med 56:124-132,1974.

11) Itano $\mathrm{S}$, Sakai $\mathrm{T}$, Ijuin $\mathrm{H}$, et al. Three cases of renal angiomyolipoma Intern Med 36:206-210, 1997.

12) Takeyama M, Arima M, Sagawa S, Sonoda T. Preoperative diagnosis of coincident renal cell carcinoma and renal angiomyolipoma in nontuberous sclerosis. J Urol 128: 579-581, 1982.

13) Lowe BA, Brewer J, Houghton DC, Jacobson E, Pitre T. Malignant transformation of angiomyolipoma. J Urol 147:1356-1358, 1992.

14) Nonomura A, Mizukami Y, Kadoya M. Angiomyolipoma of the liver. A collective review. J Gastroenterol 29: 95-105, 1994.

15) Compton WR, Lester PD, Kyaw MM, Madsen JA. Abdominal angiographic spectrum of tuberous sclerosis. Am J Roentgenol 126:807813,1976.

16) Perov ML, Gray PT. Mesenchymal hamartomas of kidney. J Urol 33: 240-261, 1960.

17) Roberts JL, Fishman EK, Hartman DS, Sanders R, Goodman Z, Siegelman SS. Lipomatous tumors of the liver: Evaluation with CT and US. Radiology 158: 613-617,1986.

18) Chan JKC, Tsang WYW, Pau MY, Tang MC, Pang SW, Fletcher CDM. Lymphangiomyomatosis and angiomyolipoma: closely related entities characterized by hamartomatous proliferation of HMB-45-positive smooth muscle. Histopathology 22:445-455,1993. 